\title{
Programa de promoción de la parentalidad positiva en la escuela: un estudio preliminar en un contexto de vulnerabilidad social
} Program to Promote Positive Parenthood at School: A Preliminary Study in a Socially Vulnerable Context Jael Vargas-Rubilar $\bowtie$, María Cristina Richaud ${ }^{2}$, Laura Beatriz Oros $^{3}$

${ }^{1}$ Consejo Nacional de Investigaciones Científicas y Técnicas, Argentina; Universidad Adventista del Plata ${ }^{2}$ Consejo Nacional de Investigaciones Científicas y Técnicas, Argentina; Universidad Adventista del Plata

${ }^{3}$ Consejo Nacional de Investigaciones Científicas y Técnicas, Argentina; Universidad de la Cuenca del Plata, Universidad Adventista del Plata; ISAM

Cervantes 70. CP.3103. Libertador San Martín, Entre Ríos, Argentina. Correo electrónico: psicojael@gmail.com

Recibido: 12 de octubre del 2017 Aprobado: 16 de febrero del 2018 Disponible en línea: 1 de abril de 2018

Cómo citar este artículo: Vargas-Rubilar, J. A., Richaud, M. C., y Oros, L. B. (2018). Programa de promoción de la parentalidad positiva en la escuela: un estudio preliminar en un contexto de vulnerabilidad social. Pensando Psicología 14(23). doi: https://doi.org/10.16925/pe.v14i23.2265

\section{Resumen}

Introducción: la parentalidad positiva requiere la conjunción equilibrada de diversos factores indispensables, tales como el apego, el afecto, la comunicación, la disciplina y la autonomía otorgada a los hijos.

Objetivo: este estudio exploró el impacto de un programa de fortalecimiento parental desde la valoración de las madres participantes y de sus hijos.

Metodología: el programa se implementó de manera grupal por medio de encuentros expositivo-participativos en una escuela de contexto socialmente vulnerable. Luego de su aplicación, se realizaron con las madres entrevistas semiestructuradas; además, se evaluaron el apego y el estilo parental percibidos por sus respectivos hijos antes y después del programa, y se compararon con un grupo control.

Resultados: se encontró que los talleres, según lo informaron las participantes, aportaron información novedosa que contribuyó a su crecimiento personal y a la sensibilidad parental, produjo un cambio en las creencias respecto a la crianza, una mejoría en la expresión del afecto, la comunicación verbal y la disciplina, significó una importante fuente de apoyo social y activó su interés en la situación escolar de sus hijos, lo cual mejoró — en algunos casos - la comunicación entre las madres y la escuela. Por otra parte, la percepción de los hijos sobre el apego y el estilo de crianza fue mejor después de la intervención con las madres, y fue mayor que la observada en el grupo control.

Conclusiones: el programa de intervención tuvo un impacto favorable en diferentes aspectos maternos que propician el ejercicio de una parentalidad positiva.

Palabras clave: intervención, eficacia/impacto, parentalidad positiva, vulnerabilidad social. 


\title{
Program to Promote Positive Parenthood at School: A Preliminary Study in a Socially Vulnerable Context
}

\begin{abstract}
Introduction: Positive parenting requires the balanced combination of various essential factors, such as attachment, affection, communication, discipline and autonomy granted to children.

Objective: This study explored the impact of a parental strengthening program based on the assessment of participating mothers and their children.

Method: The program was implemented in groups through expository-participative meetings at a school in a socially vulnerable context. After its application, semi-structured interviews with the mothers were conducted; in addition, the attachment and parental style perceived by their respective children before and after the program were evaluated and compared with a control group.

Results: We found that the workshops, as reported by the participants, provided novel information that contributed to their personal growth and parental sensitivity; produced a change in beliefs regarding parenting and an improvement in the expression of affection, verbal communication and discipline; was an important source of social support; and activated their interest in the school situation of their children, which improved - in some cases - communication between mothers and the school. Moreover, the children's perception of attachment and parenting style was better after the intervention with the mothers and better than that observed in the control group.

Conclusions: It is concluded that the intervention program had a favorable impact on different maternal aspects that encourage the exercise of positive parenthood.
\end{abstract}

Keywords: intervention, effectiveness/impact, positive parenthood, social vulnerability.

\section{Programa de promoção da parentalidade positiva na escola: um estudo preliminar em um contexto de vulnerabilidade social}

\section{Resumo}

Introdução: a parentalidade positiva requer a conjunção equilibrada de diversos fatores indispensáveis, tais como 0 apego, o afeto, a comunicação, a disciplina e a autonomia outorgada aos filhos.

Objetivo: este estudo explorou o impacto de um programa de fortalecimento parental a partir da valorização das mães participantes e de seus filhos.

Metodologia: o programa foi implementado de maneira coletiva por meio de encontros expositivo-participativos em uma escola de contexto socialmente vulnerável. Depois da aplicação, foram realizadas entrevistas semiestruturadas com as mães. Além disso, 0 apego e o estilo parental percebidos por seus respectivos filhos antes e depois do programa foram avaliados e comparados com um grupo de controle.

Resultados: de acordo com o que as participantes informaram, constatou-se que as oficinas trouxeram novas informações que contribuíram para o crescimento pessoal e para a sensibilidade parental; produziram uma mudança nas crenças com relação à criação, uma melhoria na expressão de afeto, na comunicação verbal e na disciplina; significaram uma importante fonte de apoio social e ativaram o interesse na situação escolar de seus filhos, o que melhorou - em alguns casos - a comunicação entre as mães e a escola. Por outro lado, a percepção dos filhos quanto ao apego e ao estilo de criação foi melhor depois da intervenção com as mães e foi maior do que a percepção observada no grupo de controle.

Conclusões: conclui-se que o programa de intervenção teve um impacto favorável em diferentes aspectos maternos que propiciam o exercício de uma parentalidade positiva.

Palavras-chave: eficácia/impacto, intervenção, parentalidade positiva, vulnerabilidade social. 


\section{Introducción}

Los programas de apoyo a la familia se han definido como aquellos que proporcionan sostén emocional, instrumental e informacional con el objetivo de promover el desarrollo humano, fortalecer los recursos psicosociales de los adultos en su rol de padres o cuidadores, y prevenir problemas infantiles (Gómez y Haz, 2008). Dentro de los programas de apoyo familiar, pueden incluirse numerosas modalidades y estrategias de intervención psicosocial dirigidas al refuerzo y la educación de la familia (Gracia, 1997; 2011).

Estas diferentes modalidades se focalizan en la prevención primaria y secundaria de problemas familiares, y están orientadas a identificar las fortalezas y las potencialidades de estos grupos sociales (Gracia, 1997). Al respecto, algunas investigaciones han demostrado que el apoyo social externo y la formación brindada a los padres en estos programas aumentan la autoconfianza y autoeficacia parental (e.g. Díez et al., 2016; Peña, Máiquez y Rodrigo, 2014), así como la competencia para interactuar positivamente con los hijos y favorecer su desarrollo emocional y social (e. g. Díez et al., 2016; Gómez y Haz, 2008; Gómez, Cifuentes y Ortún, 2012; Rodrigo y Byrne, 2011; Rodrigo, Byrne y Álvarez, 2016; Sanders y Morawska, 2010; Torío-López, Peña-Calvo y García-Pérez, 2015).

En lo que respecta a los resultados en los hijos, algunos programas como la "triple $\mathrm{P}$ " (Positive parenting program) han encontrado cambios favorables en el estilo de crianza y en el apego seguro percibidos por los hijos posintervención (e. g. Fujiwara, Kato y Sanders, 2011; Gómez, Muñoz y Santelices, 2008; Kotliarenco, Gómez, Muñoz y Armijo, 2009; Sanders y Morawska, 2010).

Estos programas cobran relevancia principalmente para aquellas familias que necesitan de mayor apoyo en su tarea de crianza, como son, entre otras, las que viven en contextos socialmente vulnerables, las cuales presentan determinadas características psicológicas y sociales (e. g. Gómez, Muñoz y Haz, 2007; Hidalgo-García, Lorence-Lara, Pérez-Padilla y Menéndez-Álvarez- Dardet, 2012; Oros y VargasRubilar, 2012; Richaud, 2013; Richaud y AránFilippetti, 2015). La mayoría de las investigaciones mencionan diversos factores de riesgo que se retroalimentan y pueden agruparse de la siguiente manera:

- Factores intrínsecos. Historias de vida y contexto desfavorable cotidiano que traen aparejadas indefensión, aislamiento, desesperanza, baja autoestima y autoeficacia infantil, violencia intrafamiliar, estrés parental, disminución en las competencias parentales, depresión y adicciones, entre otras problemáticas (Cirillo, 2012; Gómez et al., 2007; Lecannelier, Flores, Hoffmann y Vega, 2010; Lemos, 2009; Richaud, 2013).

- Factores extrínsecos. Precariedad de las viviendas, hacinamiento, contaminación ambiental, dificultad en la inserción laboral o desempleo, fragilidad de los vínculos familiares, barrios peligrosos, escaso acceso a servicios de salud y educación, pobreza, etc. (Kotliarenco, Cáceres y Fontecilla, 1997; Richaud, 2013).

Particularmente, en lo que respecta a la relación familia-escuela, el pacto "escuela-familia" constituye una declaración implícita acerca de lo que cada actor puede ofrecer y esperar del otro, en una tarea conjunta cuyo propósito es asegurar la continuidad del modelo de cultura y sociedad. La relación "escuela-familia" es asimétrica, ya que se plantea desde la escuela porque a esta le interesa que sus esfuerzos sean adecuadamente complementados por la familia. Paralelamente, la familia espera que los aprendizajes escolares sean también para la vida (Navarro, 2004).

En la sociedad actual, en general, pero especialmente en las familias en situación de vulnerabilidad social, existe una brecha entre familia y escuela que configura cierto déficit de educabilidad y se debe muchas veces a la rigidez de los marcos escolares. Por otra parte, la familia comienza a recibir críticas por parte de la escuela cuando esta advierte que los efectos de la socialización primaria y de la interacción del niño con otros pares o adultos fuera de ella no son siempre funcionales a los propósitos de la escuela. Es un escenario en que el pacto "escuela-familia" está en cuestión. La familia, por su parte, critica a la escuela al demandarle mayor eficacia y una oferta complementaria que incluya al menos alimentación y un ambiente seguro. Para las familias de contextos de vulnerabilidad social, la escuela tiene un "significado expresivo" más que instrumental, es decir, la experiencia escolar es importante en el presente, pues provee un clima protegido y aparta a los niños de las "malas juntas" y la droga, al tiempo que les provee un espacio de convivencia regulada por adultos responsables (Navarro, 2004).

En esta dirección, algunos estudios recientes (e. g. Tezón, 2015) han señalado una baja implicación e interés de estas familias en la educación de 
sus hijos, así como una marcada distancia sociocultural entre ambas instituciones sociales (familia y escuela), lo cual repercute en las prácticas docentes, en el desempeño de los niños y en la comunicación entre padres, docentes y directivos. Algunas realidades como las que se han descrito han impulsado la implementación de iniciativas de intervención para mejorar la relación familia-escuela en diferentes contextos (Cano y Casado, 2015; Díez y Terrón, 2006). Sin embargo, en Argentina no se han encontrado antecedentes de su aplicación como parte inherente a los programas de intervención parental en contextos de vulnerabilidad social.

Es importante destacar que algunos de los factores de riesgo mencionados se encuentran en diversos grupos sociales, no solo en aquellos de estrato socioeconómico bajo. Sin embargo, cuando estas características se plantean en un escenario caracterizado por la extrema pobreza, tienden a cronificarse (Gómez y Haz, 2008).

Muchos abordajes realizados en estos grupos sociales han evidenciado resultados alentadores con respecto a una mejoría en la parentalidad social (e.g. Bartau-Rojas y Caba-Collado, 2009; Graaf, Speetjens, Smit, de Wolff y Tavecchio, 2008; Peña, Máiquez y Rodrigo, 2014; Richaud, 2013). Las intervenciones que han mostrado cambios positivos en los beneficiarios se han basado principalmente en: (a) el enfoque de la parentalidad positiva (Rodrigo, Maiquéz y Martin, 2010), (b) el apoyo social en la intervención comunitaria (Gracia, 1997), y (c) los principios de la resiliencia familiar y parental (Barudy y Dantagnan, 2010; Walsh, 2004).

El programa de intervención familiar cuyo impacto evaluaremos en el presente estudio tiene características que lo hacen novedoso con respecto a los anteriormente mencionados: (a) se realizó sistemáticamente en un ámbito natural (la escuela) con el que se relacionan las familias, (b) se basa en un modelo de intervención educativo y comunitario (Maiquéz-Chaves y Capote-Cabrera, 2001), (c) forma parte de un programa mayor que promueve otros recursos psicológicos en niños de contextos escolares de vulnerabilidad social (Richaud, 2013), (d) ha sido pensado desde el enfoque de la parentalidad positiva (Rodrigo, Maiquéz, y Martin, 2010) basado en la atención primaria de la salud (esto es, actividades de prevención, promoción de la salud y atención familiar), lo que es poco usual en Argentina, y (e) procura mejorar la interacción entre la familia y la escuela. En este marco, el objetivo del presente trabajo es analizar los efectos preliminares de un programa de intervención parental desarrollado en un contexto escolar, al estudiar si desde la perspectiva de las madres participantes se han producido cambios positivos en el ejercicio de su parentalidad en su interacción con la escuela y en su percepción de las redes de apoyo social. Además, se analizarán los efectos indirectos de dicha intervención mediante la indagación de si se ha modificado la percepción que tienen los hijos acerca del estilo de relación y el apego materno. Se hipotetiza que luego de la intervención, las madres percibirán cambios favorables en el ejercicio de su parentalidad, en su relación con la escuela y en el apoyo social formal e informal percibido. Asimismo, los hijos percibirán un estilo de crianza y apego más positivos. Finalmente, se espera encontrar diferencias significativas en estas percepciones entre los hijos de quienes participaron del programa y los niños del grupo control, de manera que sean estas diferencias a favor del grupo experimental.

\section{Método}

Se realizó un estudio con diseño mixto (cualitativo y cuantitativo) de tipo concurrente, debido a que se aplicaron ambos métodos de investigación de forma simultánea (Hernández-Sampieri, FernándezCollado y Baptista-Lucio, 2014). A fin de analizar el impacto del programa de intervención desde la perspectiva de las madres participantes se utilizó la estrategia de análisis cualitativo, en tanto que para estudiar el efecto indirecto de la intervención sobre la percepción infantil de parentalidad (estilo de relación y apego) se trabajó con un diseño cuantitativo "antes-después" con grupo de control.

\section{Participantes}

\section{Madres}

Inicialmente, se realizó una evaluación diagnóstica de 90 familias de una cohorte de niños que concurrían a una escuela socialmente vulnerable de Entre Ríos, Argentina. Los grupos familiares pertenecían al estrato social de nivel 5 (pobreza extrema) en un $28,9 \%$, y en un $71,1 \%$ al nivel 4 (pobreza relativa), según la clasificación de la escala Graffar (Méndez-Castellano y Méndez, 1994). Debido a que el programa de intervención parental se realizó en un contexto natural (la escuela) y no fue obligatorio, en la medida en que no respondía a ninguna denuncia o judicialización familiar, la asistencia al programa 
fue irregular. Así, participaron asistemáticamente un total de 60 padres y madres. Esta condición condujo a que para el análisis de los resultados se seleccionara intencionalmente solo un grupo de 30 madres de edades comprendidas entre los 25 y 47 años $(M=38,58$; $D E=6,99$ ), que cumplieron con los siguientes criterios de inclusión: 1. Ejercer la maternidad en contextos de vulnerabilidad social de Paraná, provincia de Entre Ríos, Argentina, caracterizados como de niveles 4 (pobreza relativa) y 5 (pobreza crítica) según la escala Graffar (Méndez-Castellano y Méndez, 1994); 2. Haber participado de la intervención de fortalecimiento parental, con una asistencia no menor al 65\% de las sesiones; y 3. Cabe mencionar que, si bien fueron convocados a participar de la intervención tanto padres como madres, solo las progenitoras asistieron a los encuentros de manera frecuente.

\section{Hijos}

Se seleccionó intencionalmente un grupo experimental de 30 niños de siete y ocho años de edad, hijos de las madres que participaron del programa de intervención. Se tomó como principal criterio de inclusión que las madres de los niños hubieran participado por lo menos en un $65 \%$ de la intervención parental. El grupo de control estuvo integrado por 30 niños con las mismas características socioevolutivas del grupo experimental, cuyos padres no participaron de la intervención.

\section{Programa de intervención}

El programa se denominó "Un puente entre la familia y la escuela", debido a que uno de los principales objetivos fue fortalecer la relación entre la familia y la institución educativa (Richaud, 2013; Vargas-Rubilar, Lemos y Richaud, 2017; Vargas-Rubilar y Oros, 2011). Dicha intervención integró elementos de los modelos de intervención familiar educativo y comunitario (Máiquez-Chaves y Capote-Cabrera, 2001), ya que se implementó de forma grupal mediante encuentros expositivo-participativos y en coordinación con la escuela a la que asisten los hijos de los participantes. Estos encuentros de fortalecimiento parental se plantearon como espacios de intercambio de experiencias, vivencias y conocimientos entre los profesionales y los padres asistentes.

La agenda temática se planificó en función de los contenidos sugeridos por los padres e interventores, la información referida a la intervención realizada con los niños y otros tópicos propuestos en experiencias previas de estudios específicos de intervención con padres. Así, los tópicos abordados fueron divididos en: (a) temas sugeridos por padres e interventores: apego, empatía parental, expresión adecuada del afecto, disciplina y comunicación positiva, resiliencia familiar, parental e infantil, redes de apoyo social a familias y vinculación familia-escuela; y (b) contenidos abocados a la promoción de recursos psicológicos en padres e hijos: autoestima, emociones positivas, recursos cognitivos, conducta prosocial y habilidades sociales (ver Vargas et al., 2017; Vargas-Rubilar y Oros, 2011).

Las principales técnicas de intervención utilizadas fueron: 1. Exposición de información: consiste en brindar conocimiento teórico-práctico relevante a los padres acerca de la crianza y el desarrollo infantil; 2. Retroalimentación con pares y correflexión: se refiere a una instancia de reflexión en la que los padres e interventores intercambian y analizan sus conocimientos, creencias, actitudes y prácticas frente a situaciones cotidianas en la relación con sus hijos; 3 . Modelado de actitudes y comportamientos alternativos: consiste en la presentación de conductas alternativas a las consideradas inadecuadas en la relación paternofilial cotidiana a través de role playing durante los encuentros. Se abordaron principalmente actitudes y conductas relatadas por las participantes referidas a dificultades en la expresión del afecto, la autonomía otorgada y la disciplina aplicada a los hijos; 4. Asignación de tareas y propuestas de acción: se refiere a actividades prácticas realizadas y supervisadas durante los encuentros con el objetivo de consolidar y promover la aplicación en el ámbito familiar de algunas de las estrategias utilizadas en la escuela para fortalecer los recursos en los niños y padres; 5. Entrevistas posteriores a los sucesivos encuentros: esta instancia brindó la posibilidad de que los participantes se entrevistaran individualmente con los profesionales en los días posteriores a la reunión grupal. Estas entrevistas tuvieron el propósito de responder consultas personales difíciles de atender en las sesiones grupales, especialmente por motivos éticos y de tiempo.

\section{Instrumentos}

La percepción de las madres se indagó una vez finalizada la intervención mediante entrevistas semiestructuradas que fueron llevadas a cabo por dos psicólogas. Durante las entrevistas, se realizaron preguntas dirigidas a explorar: (a) los beneficios percibidos de la 
intervención (personales, parentales y en la relación familia-escuela), (b) los motivos atribuidos a la inasistencia de algunos padres, y (c) la preferencia del tipo de convocatoria a los encuentros. Las preguntas realizadas a las participantes, de acuerdo con la clasificación de Mertens (2005), fueron de opinión; por ejemplo: “¿n qué le parece que le han ayudado los talleres para padres?”. A partir de las respuestas de los padres podían surgir nuevas preguntas no previstas con anterioridad.

A fin de analizar el impacto del programa luego de tres meses de su finalización, se realizó una entrevista grupal (Escobar y Bonilla- Jiménez, 2011) a las participantes. En dicha entrevista se indagó con una única pregunta: ¿qué beneficios habían percibido las madres durante los meses posteriores a la finalización del programa?

Para evaluar el impacto indirecto que la intervención con los padres había generado en los niños, se analizaron los cambios en la calidad de la relación entre madres e hijos (pre y posintervención). Para esto se administraron a los hijos de las participantes y a un grupo de control de la misma escuela los siguientes instrumentos:

- El cuestionario de percepción de estilos de relación con sus padres en niños y niñas de 6-8 años de Richaud de Minzi (2002). Es una adaptación del "Children's Report of Parental Behavior Inventory" (CRPBI) desde el modelo de Schaefer (1965). Esta escala presenta 20 enunciados tanto para la madre como para el padre. La prueba mide tres dimensiones de los estilos parentales: aceptación, control patológico y autonomía extrema. De acuerdo con los datos reportados por la autora (Richaud, 2002), la consistencia interna del inventario refleja valores adecuados teniendo en cuenta la edad de los niños $(\alpha=0,65$ y 0,69 en las versiones padre y madre, respectivamente).

- La adaptación argentina (Richaud de Minzi, 2006) de la Kerns Security Scale (Kerns, Tomich, Aspelmeier y Contreras, 2000). Este autoinforme está compuesto por enunciados que miden la confianza y la disponibilidad que los niños perciben que tienen con sus padres o cuidadores. El análisis reportado por la autora para la consistencia interna por factores mostró valores satisfactorios: Confianza ( $\alpha=0,70$ para las madres, y 0,69 para los padres) y Disponibilidad ( $\alpha=0,71$ para las madres y 0,70 para los padres) (Richaud de Minzi, 2006).

\section{Procedimientos}

\section{Procedimiento de intervención y recolección de datos}

Durante la instancia de entrevistas a las 90 familias iniciales se informaron las características que asumiría el programa de intervención para padres y se consultó qué temas serían de su interés y necesidad, a fin de incluirlos en la agenda temática de las sesiones. En esta misma instancia se administró la escala Graffar (Méndez-Castellano y Méndez, 1994) para evaluar el estrato social al que pertenecían las familias.

Los temas prioritarios de abordaje sugeridos por los padres fueron cuidadosamente analizados por el equipo de investigación y, con base en esto, se diseñó en el transcurso de los siguientes dos meses la programación y los materiales de trabajo (estos fueron: coordinación con los directivos y maestros de la escuela, elección del lugar de reunión, modalidad de convocatoria, cuadernillos y protocolos de evaluación, entre otros).

La invitación a los padres para participar en la intervención se realizó a través de notas escritas en los cuadernos de comunicaciones de los niños y mediante carteles que se pegaron en la entrada de la escuela.

La intervención se realizó con una frecuencia mensual en el primer año y quincenal en el segundo, a fin de que en las semanas posteriores al taller los padres pudieran entrevistarse individualmente con el equipo. La coordinación de los encuentros la llevó a cabo un equipo interdisciplinario (psicólogos y psicopedagogos). En el marco del programa mencionado se realizó un total de 30 encuentros de 60 a 90 minutos cada uno, asociados a la concurrencia de los padres a retirar los boletines de calificaciones y otras actividades escolares de sus hijos.

Tres semanas después de finalizada la intervención se inició la sesión de evaluación a las madres. Las participantes fueron debidamente informadas en una reunión acerca de los objetivos, el método y la confidencialidad de las respuestas, así como de la libertad para retirarse de la investigación si así lo deseaban. A continuación, firmaron el consentimiento informado quienes aceptaron participar y cumplían con los criterios de inclusión del estudio. Las entrevistas se realizaron individualmente en la escuela y se grabaron en todos los casos, previa autorización de las madres. La duración de cada entrevista osciló entre 60 y 90 
minutos en función de la extensión de las respuestas de las participantes. Tres meses después de finalizada la aplicación del programa se realizó una entrevista grupal con el fin de valorar los beneficios percibidos por las participantes pasado ese tiempo.

Por su parte, la evaluación de los niños, tanto pre como posintervención, fue individual y se realizó en la escuela durante los horarios habituales de clases, manteniendo así las condiciones estandarizadas. Previamente a la administración de las pruebas se solicitó la autorización a directivos y docentes. Asimismo, se solicitó el consentimiento informado de los padres o tutores como requisito para la participación de los niños.

Además, cabe resaltar que previo a la realización de la investigación al proyecto lo evaluaron dos comités de ética: 1. El comité del Centro Interdisciplinario de Investigaciones en Psicología Matemática y Experimental; y 2. El Comité de la Universidad Adventista del Plata.

\section{Procedimiento de análisis de la información}

Las respuestas de las participantes a las entrevistas individuales fueron transcritas y sistematizadas con el fin de efectuar posteriormente un análisis de contenido (Krippendorff y Wolfson, 1990). Las respuestas a las entrevistas se compararon con el propósito de buscar categorías comunes y no comunes hasta no producirse más información adicional. Para analizar la confiabilidad de las categorías establecidas se calculó el grado de acuerdo entre las categorizaciones independientes de dos investigadoras con experiencia en la temática, a través del coeficiente Kappa de Cohen. La sistematización final se realizó por medio del programa para análisis de datos cualitativos Atlas.ti 6.

Por otra parte, los datos cuantitativos evaluados en los dos grupos de niños se ingresaron a una planilla del Statistical Package for the Social Sciences (sPss) versión 22.0. Dado que las variables presentaron una distribución normal, se decidió utilizar la prueba $t$ para el contraste de medias. A fin de analizar la relación con sus progenitoras y el apego percibidos en los hijos de las madres participantes antes y después de la intervención, se realizaron pruebas t para muestras relacionadas. Con la finalidad de comparar los resultados obtenidos en el grupo con intervención y el grupo control sin intervención, se realizaron pruebas t para muestras independientes.

\section{Resultados}

A continuación, se presentan los resultados referidos a los temas centrales que se indagaron en las madres participantes del programa. Asimismo, se presentan los resultados obtenidos sobre la calidad de la relación entre madres e hijos desde la perspectiva de los niños.

\section{Beneficios de la intervención desde la perspectiva de las madres}

A partir de las respuestas de las madres con respecto a los beneficios de la intervención en el ámbito personal y parental, así como en su vinculación con la escuela, se identificaron las siguientes categorías: 1 . Información novedosa, es decir, el reconocimiento e incorporación de conocimiento nuevo acerca de la crianza de los hijos. Por ejemplo: "Para mí ha sido muy importante, porque he descubierto cosas nuevas que de otra manera no sabría" (E: 1, comunicación personal, 9 de septiembre de 2013), ${ }^{1}$ o "A mí me ayudó aclarar ciertas dudas, saber más de los hijos" (E: 5, comunicación personal, 12 de septiembre de 2013 ); 2. Crecimiento personal en relación con el rol materno: “Me ayudó para mí misma también porque antes me pasaba el día encerrada en mi casa, aprendí mucho como madre..." (E: 4, comunicación personal, 12 de septiembre de 2013), o "Me ha ayudado a madurar más como mamá” (E: 6, comunicación personal, 12 de septiembre de 2013); 3. Sensibilización parental en relación con la conducta de los hijos, implicación en sus necesidades y sintonía con sus emociones (empatía parental): "A mí me ha ayudado a tratarlos mejor, como si fueran niños. Tenés que bajar el nivel” (E: 1, comunicación personal, 9 de septiembre de 2013), o "A mí me gustaba muchísimo por eso yo venía. Me servía a estar más atenta a ellos, a lo que piensan y quieren"; 4. Cambios en las creencias acerca de la crianza, es decir, cambios en lo que piensan (actitudes, ideas y percepciones) respecto a la formación de los hijos, como por ejemplo: "Me ayudó a pensar de otra manera" (E: 1, comunicación personal, 9 de septiembre de 2013), o "Me ayudó a tratar a los más pequeños, no sabía bien cómo tratar a los niños" (E: 5, comunicación personal, 12 de septiembre de 2013), "Me han ayudado las charlas, los consejos...

\footnotetext{
Nota. La simbología utilizada en los resultados es la siguiente: E: entrevista. El número arábigo equivale al número de la entrevista (e. g. 5).
} 
cuando se decía cómo llevar algunas situaciones por distintas etapas" (E: 3, comunicación personal, 10 de septiembre de 2013); 5. Cambios en la expresión del afecto: "Aprendí a no sentir vergüenza de darles besos, abrazos y muchos te quieros" (E: 4, comunicación personal, 12 de septiembre de 2013), "Yo creo que me ayudó a expresar el cariño a mis hijos, porque a mí no me dieron cariño" (E: 7, comunicación personal, 13 de septiembre de 2013 ); 6. Cambios en la disciplina aplicada: "Siento que los tengo más encaminados, que tengo el control” ( $\mathrm{E}: 3$, comunicación personal, 10 de septiembre de 2013), o "Me enseñaron muchas cosas, a no gritarles a los chicos. A veces por no pegarles les gritaba" (E: 7, comunicación personal, 13 de septiembre de 2013); 7. Cambios en la comunicación: "Aprendí sobre la disciplina para lograr un vínculo más comunicativo" (E: 4, comunicación personal, 12 de septiembre de 2013), "Cuando pasan cosas, algo simple como grave, me acuerdo de cosas que hemos hablado acá, lo veo y le trato de decir las cosas de otra manera" (E: 1 , comunicación personal, 10 de septiembre de 2013); 8. Apoyo social, es decir, la percepción del soporte recibido por parte de las redes sociales a nivel emocional e informacional (incluye orientación, consejo y guía): (a) apoyo social informal o de grupos primarios como lo son miembros de la familia, vecinos, otras madres del taller, etc., como, por ejemplo, "Escuchás lo que dicen otros padres, lo podés poner o intercambiar mi vida y tu vida, por ahí las escucho a las otras mamás y es bueno, a mí me ayuda"(E: 8, comunicación personal, 13 de septiembre de 2013), "He afianzado mi relación con otras mamás" (E: 3, comunicación personal, 10 de septiembre de 2013), y (b) apoyo social formal, es decir, profesional, por ejemplo, "Yo necesitaba hablar con alguien como ustedes de lo que me estaba pasando.... la relación de uno con los hijos, los hijos y la escuela" (E: 6, comunicación personal, 12 de septiembre de 2013), "Escuchar las historias de otros padres que nos contás, ayuda" (E: 2, comunicación personal, 10 de septiembre de 2013), "Me ha ayudado charlar, contar cosas que a otras personas no les cuento, es un desahogo...” (E: 8, comunicación personal, 13 de septiembre de 2013); y 9. Mejor relación con la escuela, es decir, que la relación con la escuela mejoró después de la intervención: "Creo que mejoró porque hablo más con las maestras y los directores" (E: 7, comunicación personal, 12 de septiembre de 2013), "Antes hablaba con la gente de la escuela lo justo y necesario, ahora vengo más a la escuela" (E: 8 , comunicación personal, 12 de septiembre de 2013). Asimismo, en esta categoría emergieron opiniones divergentes que se clasificaron como igual relación con la escuela, es decir, la relación con la escuela no se modificó después de la intervención: "La relación con la escuela siempre fue igual, sigue siendo lo mismo... "(E: 2, comunicación personal, 10 de septiembre de 2013), o "Para mí es igual, es como que esto no tiene que ver con la escuela" (E: 4, comunicación personal, 12 de septiembre de 2013).

A pesar de que no formó parte de los objetivos principales del estudio, pero entendiendo que la información podría resultar de gran utilidad en el propósito de mejorar el alcance y la eficacia de la intervención en aplicaciones futuras, se decidió indagar la opinión de las madres con respecto a las razones de inasistencia de otros padres y madres al programa de intervención, así como las mejores modalidades de convocatoria para este tipo de actividades con el fin de aumentar la concurrencia.

Las madres entrevistadas distinguieron los siguientes motivos por los que otros padres no asisten a los talleres: 1 . Por percepción de bajo beneficio: "Muchos padres sienten que no lo necesitan" (E: 1, comunicación personal, 10 de septiembre de 2013), o "Muchos padres son autosuficientes, dicen: ¿para qué voy a ir?” (E: 12, comunicación personal, 18 de septiembre de 2013); 2. Por desinterés: "Hay padres a los que nos les interesa, no les llama la atención" (E: 4, comunicación personal, 12 de septiembre de 2013); "Algunos dicen que no les interesa, pero nunca vinieron, no conocen" (E: 7, comunicación personal,13 de septiembre de 2013); y 3. Razones de trabajo o tiempo: "Algunos trabajan como yo y se les complica venir" (E: 11, comunicación personal, 18 de setiembre de 2013), o "A mí me gustaba mucho venir porque me ayudaba un montón, pero después empecé a trabajar" (E: 13, comunicación personal, 18 de septiembre de 2013).

Finalmente, cuando se les preguntó cuál sería la forma más indicada de convocar a los padres a los encuentros, las madres identificaron las siguientes modalidades: 1 . Convocatoria personal, es decir, que los interventores convoquen personalmente a los padres: "Me parece que ayudaría mucho visitarlos en los hogares. Aunque es complicado, yo sé por el trabajo que a veces es arriesgado ir a las casas en el barrio" (E: 5, comunicación personal, 12 de septiembre de 2013); 2. Convocatoria escrita: "Yo les pondría una frase clave, algo como: "Si ama a su hijo, dedique por lo menos una hora a ellos" (E: 4, comunicación personal, 12 de septiembre de 2013); 3. Convocatoria 
a través de otros padres: "Creo que puede ayudar que nosotras invitemos a más padres" (E: 7, comunicación personal, 13 de septiembre de 2013), o "Creo que la invitación de boca a boca es mejor" (E: 6, comunicación personal, 13 de septiembre de 2013); y 4. Convocatoria actual: "Creo que lo que han hecho ha sido bueno, simplemente que a veces lleva tiempo darse cuenta de lo que uno necesita" (E: 1, comunicación personal, 10 de septiembre de 2013).

En lo que respecta a la confiabilidad de los datos, los resultados mostraron un alto nivel de acuerdo entre las codificadoras (Kappa de Cohen superior a $0,80)$ en todas las categorías analizadas.

\section{Beneficios percibidos por el grupo luego de tres meses de intervención}

Cuando se les consultó a las participantes, tres meses después de la intervención, acerca de los cambios que habían percibido como grupo, las madres relataron una serie de cambios positivos en ese sentido. En dicha oportunidad, comentaron que habían realizado visitas a hospitales, habían organizado un ropero escolar (venta de ropa para juntar fondos escolares) y que se ayudaban unas a otras a llevar o recoger los niños de la escuela, compartían recetas de cocina entre ellas y se reunían con frecuencia a conversar y a tomar mate, entre otras cosas.

\section{La calidad de la relación entre madres e hijos desde la perspectiva de los niños}

Los resultados mostraron que luego de la intervención con las madres, los niños aumentaron significativamente su percepción de aceptación materna, disminuyeron la de control patológico y la de autonomía extrema. Asimismo, se observó que aumentaron significativamente la confianza y la disponibilidad maternas percibidas por los niños luego del programa (véase la tabla 1).

Como contraparte, en el grupo sin intervención tanto el estilo de crianza como el apego percibido no presentaron modificaciones significativas antes y después de la intervención (véase la tabla 2).

Tabla 1

Comparación del estilo parental y el apego percibido en los hijos de las participantes antes y después de la aplicación del programa

\begin{tabular}{ccccccc}
\hline \multirow{2}{*}{ Variable } & \multicolumn{2}{c}{ Preintervención } & \multicolumn{2}{c}{ Posintervención } & \multicolumn{2}{c}{ Estadísticos } \\
\cline { 2 - 7 } & Media & Desvío & Media & Desvío & t & p \\
\hline Aceptación & 2,49 & 0,327 & 2,87 & 0,125 & 0,26 & 0,007 \\
Control patológico & 2,06 & 0,259 & 1,54 & 0,379 & 4,42 & 0,001 \\
Autonomía extrema & 2,14 & 0,490 & 1,64 & 0,452 & 2,08 & 0,049 \\
Confianza & 1,34 & 0,335 & 2,67 & 0,366 & 7,85 & 0,000 \\
Disponibilidad & 1,74 & 0,360 & 2,29 &, 277 & $-3,36$ & 0,007 \\
\hline
\end{tabular}

Nota. Elaboración propia

Tabla 2

Comparación del estilo parental y el apego materno percibido por los niños sin intervención parental

\begin{tabular}{|c|c|c|c|c|c|c|}
\hline \multirow{2}{*}{ Variable } & \multicolumn{2}{|c|}{ Preintervención } & \multicolumn{2}{|c|}{ Posintervención } & \multicolumn{2}{|c|}{ Estadísticos } \\
\hline & Media & Desvío & Media & Desvío & $\mathrm{t}$ & $p$ \\
\hline Aceptación & 2,41 & 0,387 & 2,40 & 0,345 & 0,010 & 0,921 \\
\hline Control patológico & 1,99 & 0,340 & 2,00 & 0,353 & $-0,812$ & 0,436 \\
\hline Autonomía extrema & 2,17 & 0,308 & 2,14 & 0,353 & 0,623 & 0,547 \\
\hline Confianza & 1,49 & 0,432 & 1,50 & 0,413 & 1,00 & 0,341 \\
\hline Disponibilidad & 1,76 & 0,488 & 1,81 & 0,451 & $-1,39$ & 0,192 \\
\hline
\end{tabular}

Nota. Elaboración propia 
Debido a que todas las medidas del grupo control y experimental eran equivalentes en todas las dimensiones del estilo parental y el apego antes de la intervención $(p>0,05)$, se realizó una comparación posintervención. Así, al comparar el estilo parental y el apego percibidos entre el grupo con intervención y el grupo control se encontraron diferencias significativas a favor del grupo de hijos cuyas madres participaron de la intervención parental (véase la tabla 3).

\section{Discusión}

Existen diversos estudios que destacan los efectos positivos de los programas de intervención familiar, tanto para los padres como para los hijos. Sin embargo, la mayoría de estos programas son gubernamentales, obligatorios y dirigidos a determinadas problemáticas. Por tanto, el objetivo del presente trabajo fue analizar los resultados alcanzados por un programa de fortalecimiento parental diseñado a partir del enfoque de la parentalidad positiva, realizado en contexto natural (i. e. escolar), y focalizado en la atención primaria de la salud, desde la perspectiva de las madres participantes y de sus respectivos hijos. Con respecto a la primera hipótesis enunciada, se observó que, efectivamente, después de esta modalidad de intervención parental, las madres informaron cambios favorables en el ejercicio de su parentalidad, en su relación con la escuela y en el apoyo social formal e informal percibido. En primer lugar, las madres manifestaron sentirse más competentes en la relación con sus hijos, ya que habían recibido información novedosa para ellas que contribuyó a su crecimiento personal y a la sensibilización parental. Por otra parte, informaron cambios en sus creencias acerca de la crianza y como se sentían más capaces de expresar el afecto, podían comunicarse mejor y aplicar una disciplina más adecuada a sus hijos (estilo parental más positivo), en concordancia con lo informado por otros estudios (Bartau-Rojas y Caba-Collado, 2009; Fujiwara et al., 2011; Gómez, et al., 2012; Gómez y Muñoz, 2012; Peña et al., 2014; Sanders y Morawska, 2010; Torío-López et al., 2015).

En segundo lugar, la intervención realizada fue percibida como una importante fuente de apoyo social formal e informal para las participantes. En este sentido, es relevante enfatizar que a partir de intervenciones - no obligatorias - en situaciones naturales, dentro de la vida cotidiana del niño y de sus padres, y no enmarcadas en programas gubernamentales más formalizados, se encuentran resultados similares a los alcanzados, por ejemplo, en Chile, por Gómez et al. (2012), quienes llevaron a cabo intervenciones psicoeducativas y comunitarias durante un periodo de 12 a 18 meses, con intervenciones domiciliarias y en el marco de un programa gubernamental. Así, nuestra propuesta, al tener un objetivo de atención primaria y realizarse en el ámbito natural de la escuela, logra que estas acciones impacten sobre muchos más niños (e.g. hijos no escolarizados) que en los programas dirigidos a padres de niños con determinadas características y en situaciones particulares, lo cual permite a los participantes compartir experiencias adversas en un contexto no amenazante. La percepción de apoyo del grupo disminuye la culpabilización y promueve la motivación al cambio a través de la percepción de vivir dificultades compartidas con pares. Así, las madres y padres correflexionan sobre sus propias experiencias diarias y situaciones específicas de la vida familiar y parental para resignificarlas y encontrar alternativas más positivas en

Tabla 3

Comparación del estilo parental y el apego percibido entre los grupos con y sin intervención después de la aplicación del programa

\begin{tabular}{ccccccc}
\hline \multirow{2}{*}{ Variable } & \multicolumn{2}{c}{$\begin{array}{c}\text { Grupo con intervención } \\
(\mathbf{n}=\mathbf{3 0})\end{array}$} & \multicolumn{2}{c}{$\begin{array}{c}\text { Grupo sin intervención } \\
(\mathbf{n}=30)\end{array}$} & \multicolumn{2}{c}{ Estadísticos } \\
\cline { 2 - 7 } & Media & Desvío & Media & Desvío & $\mathrm{t}$ & $p$ \\
\hline Aceptación & 2,87 & 0,125 & 2,40 & 0,345 & 4,22 & 0,001 \\
Control patológico & 1,54 & 0,379 & 2,03 & 0,353 & $-3,13$ & 0,005 \\
Autonomía extrema & 1,63 & 0,452 & 2,14 & 0,353 & $-2,93$ & 0,008 \\
Confianza & 2,67 & 0,360 & 1,50 & 0,413 & 7,03 & 0,000 \\
Disponibilidad & 2,29 & 0,273 & 1,89 & 0,451 & 2,98 & 0,000 \\
\hline
\end{tabular}

Nota. Elaboración propia 
su vida cotidiana. De esta manera, la intervención se apoya en el aprendizaje por modelado a partir de la participación conjunta de los profesionales y los participantes (Vargas et al., 2017).

En efecto, las madres se unieron por un interés común, relativo a la situación escolar de sus hijos y a la necesidad de mejorar su parentalidad. Este objetivo común las llevó en parte a afianzarse como grupo, aun algunos meses después de finalizada la intervención, y se manifestó en su interés y preocupación por colaborar con su comunidad: hacer visitas a hospitales, organizar un ropero escolar (venta de ropa a fin de juntar fondos para la escuela), a ofrecerse ayuda para llevar o recoger los niños o a compartir recetas de cocina entre ellas, así como otras actividades. De igual forma, mencionaron que se había fortalecido el apoyo formal, específicamente por el soporte recibido de las interventoras, así como de profesionales con los que tomaron contacto a partir de la decisión, por ejemplo, de iniciar terapia psicológica. Esta determinación surgió durante el proceso de intervención como resultado de la toma de conciencia de su problemática, al mismo tiempo que de conocer y desmitificar al psicólogo y a otros profesionales de la salud.

Otro de los objetivos de la intervención fue producir un cambio favorable en la alianza familia-escuela que, como mencionan algunos autores (Rivas-Borrel y Ugarte-Artal, 2014; Tezón, 2015), se halla deteriorada por un desencuentro en las expectativas de ambas instituciones. En este sentido, la intervención activó el interés de los participantes en la situación escolar de sus hijos y en muchos casos mejoró la comunicación entre los padres, los docentes y los directivos de la escuela, la cual antes de la intervención era inexistente, tal como quedó manifestado a través de los comentarios positivos expresados por algunas madres participantes: "Creo que sí mejoró porque hablo más con las maestras y los directores" (E: 1, comunicación personal, 10 de septiembre de 2013). Sin embargo, también ocurrió que muchas madres percibieron la intervención como algo externo y no perteneciente a la escuela. Por tanto, consideramos que el objetivo de mejorar la relación familia-escuela ha sido parcialmente cumplido, especialmente por la gran complejidad que implica esta alianza. Se estima que generar otras instancias de diálogo y participación entre ambas instituciones durante la intervención podría contribuir a un mayor acercamiento entre padres, docentes y directivos (participación de los padres en actos escolares y fechas especiales, realización de actividades que integren familia y escuela, etc.).

De esta manera, a través de la intervención en la escuela y en situaciones de la vida cotidiana (por ejemplo, buscar un boletín de calificaciones o asistir a una fiesta escolar), las madres se concientizaron de necesidades que hasta el momento no habían visualizado o que habiéndolas percibido no sabían a quién recurrir para mejorarlas. En este sentido, coincidimos con Rodrigo y Byrne (2011) en que las madres en situación de riesgo social generalmente tienden a buscar apoyo al enfrentarse tanto a problemas de los hijos como a problemas personales, y que este soporte lo encontrarían en los recursos del vecindario y en la asistencia de instituciones sociales, es decir, en el exosistema (Bronfenbrenner, 1979; 1989). Otros estudios han señalado - coincidiendo con nuestros resultados- cómo después de una intervención los padres contarían con una red de apoyo más amplia (Hidalgo-García, Menéndez-Álvarez Dardet, Sánchez-Hidalgo, Lorence-Lara y Jiménez-García, 2009), en especial con redes informales de apoyo social (Rodrigo y Byrne, 2011).

Como se mencionó anteriormente, uno de los objetivos de la intervención con los padres es lograr un mejor vínculo con los hijos. Para evaluar si se produce o no, la percepción de los niños es fundamental. Más allá de lo que puedan relatar los padres, resulta particularmente importante conocer cómo han impactado estos supuestos cambios en la percepción que el niño tiene de la situación. En este sentido, y en línea con la segunda hipótesis, se encontró que los niños percibieron cambios positivos en el estilo de crianza de las madres y se sintieron más queridos, menos controlados a través del castigo, y más protegidos y contenidos después de la intervención. Al mismo tiempo, los hijos percibieron cambios en el vínculo con la madre e informaron mayor confianza en su amor y mayor disponibilidad de esta ante sus necesidades (esto es, apego más seguro). Las intervenciones en apego, principalmente las que estimulan la sensibilidad parental y la visualización de las necesidades de los hijos, impactarían positivamente en la relación vincular entre madre e hijo (Gómez et al., 2008). Es importante destacar que estos resultados coinciden con la opinión manifestada por las madres participantes con respecto a la percepción de la relación con sus hijos (cambios en expresión afectiva, comunicación y disciplina) y con lo informado por otros programas muy reconocidos, como la triple $\mathrm{P}$, que 
encontraron progresos en la parentalidad percibida por los niños (Fujiwara et al., 2011; Graaf et al., 2008).

Finalmente, un aspecto no contemplado en principio, pero surgido como necesidad a partir del alto ausentismo de algunos progenitores a los talleres, fue explorar la opinión de las madres asistentes a los encuentros con respecto a las razones de la baja adherencia por parte de otros padres, indicada por algunos estudios como un elemento clave en la evaluación de la integridad del programa (Duerden y Witt, 2012). De acuerdo con lo manifestado por las madres, las razones serían la percepción de bajo beneficio, el desinterés y la falta de tiempo por razones de trabajo. Cabe mencionar que la convocatoria se hizo extensiva tanto a padres como a madres. No obstante, generalmente asistían solo las madres y algunos padres de forma esporádica, de manera similar a lo informado por otros estudios (e.g. Bartau-Rojas y Caba-Collado, 2009).

La deserción en este tipo de intervenciones o la falta de adherencia de algunos padres puede deberse, tal como las madres participantes han informado, a creencias preconcebidas acerca de que la intervención les proporcionará un bajo beneficio. También es probable que muchos de los padres participantes no hayan tomado conciencia aún de la necesidad de un cambio en el ejercicio de su rol parental (e. g. "simplemente que a veces lleva tiempo darse cuenta de lo que uno necesita"), lo cual coincide con lo que indican otros estudios similares (Rodrigo y Byrne, 2011). En este sentido, es posible que muchas de las madres que no asisten a los encuentros aún no hayan analizado las fortalezas y debilidades de su rol materno. En consecuencia, una de las tareas pendientes es optimizar estrategias para captar el interés de estos padres (convocatoria) y revisar nuevas modalidades de intervención.

No obstante, es importante mencionar que algunos estudios (e. g. Duerden y Witt, 2012) han destacado la importancia de realizar no solo un análisis de los resultados de la intervención, sino de las características de la implementación del programa, es decir, si existieron diferencias u obstáculos en su implementación. Así, cuando se combinan ambas evaluaciones (de aplicación y de resultados) se facilita la identificación de qué aspectos se deberían optimizar en lo sucesivo.

Por esta razón, nos pareció relevante indagar como último aspecto la opinión de las madres con respecto a la forma más apropiada de convocatoria a los encuentros. Las participantes sugirieron las siguientes modalidades: presencial, escrita, a través de los padres participantes y, en mayor medida, la modalidad habitual mediante notas en los cuadernos de los niños y carteles en la escuela. En este sentido, es importante aclarar que para algunas participantes la forma de convocatoria no hace una diferencia en la asistencia, sino el interés o motivación para asistir. Otro grupo de madres considera que la convocatoria personal-presencial y a través de otras madres participantes podría aumentar la asistencia. Es posible que una convocatoria que incluya más de una modalidad pueda aumentar presencia de los beneficiarios en la intervención. En efecto, el proceso de convocatoria y asistencia de los participantes es uno de los más difíciles desafíos que presenta este tipo de programas. Debe tenerse en cuenta que muchos de los padres que no han solicitado ayuda para criar a sus hijos, solucionar problemas o mejorar los vínculos no ven la necesidad de modificar sus actitudes y conductas (Vargas et al., 2017).

\section{Proyecciones futuras y limitaciones}

En síntesis, como fortalezas del programa abierto aquí propuesto, en situación natural, continuo e integrado con otras acciones de intervención en la escuela, se han podido evidenciar cambios positivos en el conocimiento y las creencias acerca de la crianza, la sensibilidad parental, las prácticas de crianza y el apoyo social percibido. Todos estos elementos constituyen, de acuerdo con lo mencionado por especialistas en el área (Barudy y Dantagnan, 2010; Cirillo, 2012), eslabones esenciales para estructurar una parentalidad más positiva. Se estima que la promoción de estos factores mediante la intervención realizada ha resultado favorable, e incluso podría tener un impacto significativo a largo plazo en los padres e hijos participantes si se considera el importante rol de la familia en el transcurso de todo el ciclo vital (VargasRubilar, 2009). Sería interesante establecer como una política de Estado el desarrollo de este tipo de intervenciones dirigida a los grupos de padres de niños en edad escolar que concurren a establecimientos educativos iniciales y primarios.

Además, insistimos en la importancia de promover las intervenciones en contextos en los que el niño se desarrolla a diario, con el objetivo de desmitificar la intervención y hacerla menos invasiva y parte de la realidad cotidiana de las familias.

Por otra parte, como tarea pendiente se considera sumamente valioso el diseño y la aplicación de 
programas de apoyo parental también en otros contextos o estratos socioeconómicos: padres de poblaciones rurales, de estratos socioeconómicos medios y altos, y poblaciones en riesgo social de grandes ciudades (por ejemplo, Buenos Aires). Dichas intervenciones deberían contar con un diagnóstico previo que les permita ajustarse a la necesidad de cada grupo social.

No obstante lo mencionado, el abordaje aquí propuesto ostenta algunas limitaciones que se deben analizar. En primer lugar, al ser una intervención natural y voluntaria, sin premios o castigos como en general implican programas más formalizados y organizados por el Estado, ha habido una asistencia muy irregular por parte de los padres y una implicación insuficiente desde la escuela. Es necesario, más allá de los incentivos (becas, planes asistenciales, etc.) o puniciones (quita de la tenencia de los niños o hasta cárcel) que se incluyen en los programas gubernamentales, encontrar nuevas formas de motivación que deberán ser cuidadosamente planificadas como un elemento sustancial de la intervención. Si esto se logra, nos aseguraremos de que concurra la mayoría de los padres, lo que pondrá en evidencia la ausencia de algunos padres con graves problemáticas que necesitarán una asistencia especial. En este sentido, dicho tipo de abordaje permitiría como un objetivo extra la detección de situaciones altamente problemáticas.

Finalmente, nuestra propuesta de intervención y evaluación se llevó a cabo solo en un centro educativo. No obstante, es una iniciativa que responde a la necesidad de tomar en consideración la voz de los participantes (padres e hijos), con la finalidad de llevar a cabo intervenciones sociales y contextualmente relevantes. Si bien el programa se diseñó para contextos de vulnerabilidad social, el abordaje de familias de alto riesgo (esto es, con múltiples problemáticas) debería articular un abordaje más amplio (Gómez et al. 2007). Así, las familias que presenten: consumo de sustancias adictivas, enfermedad mental de los progenitores o abandono de las funciones parentales de uno o ambos padres o violencia intrafamiliar, deberían contar con intervenciones especializadas y un mayor monitoreo y seguimiento para el mantenimiento de los cambios (Gómez et al., 2008).

\section{Referencias}

Bartau-Rojas, I. y Caba-Collado, M. A. (2009). Una experiencia de mejora de las habilidades para la parentalidad y el desarrollo sociopersonal de los menores en contextos de desprotección social. Intervención Psicosocial, 18(2), 135-151.

Barudy, J. y Dantagnan, M. (2010). Los desafíos invisibles de ser madre o padre. Manual de evaluación de competencias y resiliencia parental. Barcelona: Gedisa.

Bronfenbrenner, U. (1979). The Ecology of Human Development. Cambridge, ma: Harvard University Press.

Bronfenbrenner, U. (1989). Ecological system theory. En R. Vasta (Comp.), Annals of Child Development, Vol. 6. Greeenwich: CT JAI Press.

Cano, R., y Casado, M. (2015). Escuela y familia. Dos pilares fundamentales para unas buenas prácticas de orientación educativa a través de las escuelas de padres. Revista Electrónica Interuniversitaria de Formación del Profesorado, 18(2) 1527. doi: 10.6018/reifop.18.2.219491

Cirillo, S. (2012). Malos padres: modelos de intervención para recuperar la capacidad de ser padre o madre. Buenos Aires: Gedisa.

Díez, M., Jiménez, L., López-Gaviño, F., Román, M., Oliva A., Jiménez-Morago, J. (...) y Antolín-Suarez, L. (2016). Promoción de parentalidad positiva en Polígono Sur. Diseño de un programa para familias en situación de riesgo psicosocial. Apuntes de Psicología, 34, 2-3, 113-118.

Díez, E., y Terrón, E. (2006). Romper las barreras entre la familia y la escuela. Experiencia de investigaciónacción en los centros escolares para promover la relación con las familias. Culture and Education, 18, 3-4, 283-294. doi: 10.1174/113564006779172993

Duerden, M. D. y Witt, P. A. (2012). Assessing Program Implementation: What It Is, Why It's Important, and How to Do It. Journal of Extension, 50(1). doi: 10.1016/ S0272-7358(97)00043-3

Escobar, J. y Bonilla-Jiménez, F. (2009). Grupos focales: una guía conceptual y metodológica. Cuadernos Hispanoamericanos de Psicología, 9(1), pp. 51-67.

Fujiwara, T., Kato, N., y Sanders, M. R. (2011). Effectiveness of Group Positive Parenting Program (Triple $\mathrm{P}$ ) in Changing Child Behavior, Parenting Style, And Parental Adjustment: An Intervention Study in Japan. Journal of Child and Family Studies, 20, 804-814. doi:10.1007/s10826-011-9448-1

Gómez, E., Cifuentes, B. y Ortún, C. (2012). Padres competentes, hijos protegidos: evaluación de los resultados del programa "Viviendo en Familia". Psychosocial Intervention, 21(3), 259-271. doi: 10.5093/in2012a23

Gómez, E. y Haz, A. M. (2008). Intervención familiar preventiva en programas colaboradores del Sename: la 
perspectiva del profesional. Psykhé, 17(2), 53-65. doi: $10.4067 / \mathrm{s} 0718-22282008000200005$

Gómez, E. y Muñoz, M. (2012). Efectos de la terapia de interacción guiada sobre el bienestar de díadas en riesgo psicosocial. Terapia Psicológica, 30(1), 15-24. doi: 10.4067/S0718-48082012000100002

Gómez, E., Muñoz, M. M. y Haz, A. M. (2007). Familias multiproblemáticas y en riesgo social: características e intervención. Psykhe, 16(2), 43-54. doi: 10.4067/ S0718-22282007000200004

Gómez, E., Muñoz, M. M. y Santelices, M. P. (2008). Efectividad de las intervenciones en apego con infancia vulnerada y en riesgo social: un desafío prioritario para Chile. Terapia Psicológica, 26(2), 241-251.

Graaf, I., Speetjens, P., Smit, F., de Wolff, M. y Tavecchio, L. (2008). Efectividad del programa de crianza positiva Triple $\mathrm{P}$ en problemas de conducta en los niños: Un meta-análisis. Behavior Modification, 32(5), 714-735.

Gracia, E. (1997). El apoyo social en la intervención comunitaria. Barcelona: Paidós.

Gracia, E. (2011). Apoyo social e intervención social y comunitaria En I. Fernández, J. F. Morales y F. Molero (Coords.), Psicología de la intervención comunitaria (pp. 129-171). Bilbao: Desclée de Brower.

Hernández-Sampieri, R., Fernández-Collado, C. y Baptista-Lucio, M. (2014). Metodología de la investigación. Madrid: McGraw-Hill Interamericana.

Hidalgo García, M. V., Menéndez-Álvarez-Dardet, S., Sánchez-Hidalgo, J., Lorence-Lara, B. y Jiménez García, L. (2009). La intervención con familias en situación de riesgo psicosocial: aportaciones desde un enfoque psicoeducativo. Apuntes de Psicología, 27(2-3), 413-426.

Hidalgo García, M. V., Lorence Lara, M., Pérez Padilla, J. y Menéndez- Álvarez Dardet, S. (2012). Tipología de familias en riesgo social: el papel de la estructura familiar. Revista de Mexicana de Psicología, 29(2), 165-174.

Kerns, K. A., Tomich, P. L., Aspelmeier, J. E. y Contreras, J. M. (2000). Attachment-Based Assessments of Parent-Child Relationships in Middle Childhood. Developmental Psychology, 36(5), 614-626. doi: 10.1037/0012-1649.36.5.614

Kotliarenco, M. A., Cáceres, I. y Fontecilla, M. (1997). Estado de arte en resiliencia. Santiago de Chile: Organización Panamericana de la Salud. Fundación Kellogg, Ceanim.

Kotliarenco, M., Gómez, E., Muñoz, M. y Armijo, I. (2009). Evaluación pre-post del desarrollo psicomotor y el estilo de apego en los usuarios de los centros de desarrollo infantil temprano. Summa Psicológica, 6(2), 89-104.
Krippendorff, K. y Wolfson, L. (1990). Metodología de análisis de contenido: teoría y práctica. Barcelona: Paidós.

Lecannelier, F., Flores, F., Hoffmann, M. y Vega, T. (2010). Trayectorias tempranas de la agresión: evidencias y la propuesta de un modelo preventivo. En D. Sirpolu y H. Salgado (Eds.), Trayectorias. Santiago: Universidad del Desarrollo.

Lemos, V. (2009). Características de personalidad infantil asociadas al riesgo ambiental por situación de pobreza. Interdisciplinaria, 26, 5-23.

Navarro, L. (2004). La escuela y las condiciones sociales para aprender y enseñar equidad social y educación en sectores de pobreza urbana. Buenos Aires: IIPE-Unesco.

Máiquez Chaves, M. L. y Capote-Cabrera, C. (2001). Modelos y enfoques en intervención familiar. Intervención Psicosocial, 10(2), 185-198.

Méndez-Castellano, H. y Méndez, M. C. (1994). Sociedad y estratificación, método Graffar-Méndez Castellano. Caracas: Fundacredesa.

Mertens, D. M. (2005). Research and Evaluation in Education and Psychology: Integrating Diversity With Quantitative, Qualitative, and Mixed Methods (2. ${ }^{\mathrm{a}}$ ed.). Thousand Oaks: Sage

Oros, L. B. y Vargas-Rubilar, J. (2012). Fortalecimiento emocional de las familias en contextos de pobreza. Suma Psicológica, 19(1), 69-80.

Peña, M., Máiquez, M. L. y Rodrigo, M. J. (2014). Efectos de la inclusión de contenidos de desarrollo personal en un programa de educación parental para familias en riesgo psicosocial. Anales de Psicología, 30(1), 201-210. doi:10.6018/analesps.30.1.152331

Richaud de Minzi, M. C. (2002). Inventario acerca de la percepción que tienen los niños y niñas de las relaciones con sus padres y madres: versión para 4-6 años. Revista Interamericana de Psicología, 36(1), 149-165.

Richaud de Minzi, M. C. (2006). Loneliness and Depression in Middle and Late Childhood: Its Relationship to Attachment and Parental Styles. Journal of Genetic Psychology, 167(2), 189-210.

Richaud, M. C. (2013). Contributions to the Study and Promotion of Resilience in Socially Vulnerable Children. American Psychologist, 68(1), 751-758. doi: 10.1037/ a0034327

Richaud, M. C. y Arán-Filippetti, V. (2015). Children's Cognitive Development in Social Vulnerability: An Interventional Experience. Journal of Psychology Research, 5(12), 684-692. doi: 10.17265/2159-5542/2015.12.003

Rivas-Borrel, S. y Ugarte-Artal, C. (2014). Formación docente y cultura participativa del centro educativo: 
claves para favorecer la participación familia-escuela. Estudios sobre Educación, 24, 153-168.

Rodrigo, M. J., Byrne, S. y Álvarez, M. (2016). Interventions to Promote Positive Parenting in Spain. En M. Israelashvili y J. L. Romano (Eds.), Cambridge handbook of international prevention science (pp. 929-956). Camdridge, RU: Cambridge University Press.

Rodrigo, M. J, Máiquez, M. L., y Martín, J. C. (2010). Parentalidad: favorecer el ejercicio de las responsabilidades parentales desde las corporaciones locales. Madrid: Federación Española de Municipios y Provincias.

Rodrigo, M. J. y Byrne, S. (2011). Apoyo social y agencia personal en madres en situación de riesgo. Intervención psicosocial, 20(1), 13-24. doi:10.5093/in2011v20n1a2.

Sanders, M. R. y Morawska, A. (2010). ¿Es posible que el conocimiento de los padres, las competencias y expectativas disfuncionales, $y$ la regulación emocional mejoren los resultados de los niños? En R. E. Tremblay, R.G. Barr, R. DeV. Peters y M. Boivine (Eds.), Enciclopedia sobre el desarrollo de la primera infancia (pp. 1-13). Montreal, Quebec: Centre of Excellence for Early Childhood Development. Recuperado de http:// www.enciclopedia-infantes.com/documents/SandersMorawskaESPxp.pdf.
Schaefer, E. S. (1965). Children's Report of Parental Behavior: An Inventory. Child Development, 36, 417-424. doi: $10.2307 / 1126465$

Tezón, M. (2015) Creencias sobre la relación familia-escuela en maestras de niños en situación de vulnerabilidad socio-económica de Buenos Aires. Hexágono Pedagógico, 6(1), 1-32. doi: 10.22519/2145888X.654

Torío-López, S., Peña-Calvo, J. V. y García-Pérez, O. (2015). Parentalidad positiva y formación experiencial: análisis de los procesos de cambio familiar. Multidisciplinary Journal of Educational Research [Remie], 5(3), 296-315.

Vargas-Rubilar, J. (2009). Percepción de clima social familiar y actitudes ante situaciones de agravio en la adolescencia tardía. Interdisciplinaria, 26(2), 289-316.

Vargas-Rubilar, J. y Oros, L. (2011). Una propuesta de intervención psicoeducativa para promover la autoestima infantil. Revista Acta Psiquiátrica y Psicológica de América Latina, 57(3), 235-244.

Vargas-Rubilar, J., Lemos, V. y Richaud, M. C. (2017) Programa de fortalecimiento parental en contextos de vulnerabilidad social: una propuesta desde el ámbito escolar. Interdisciplinaria, 34(1), 157-172.

Walsh, F. (2004). Resiliencia familiar: estrategias para su fortalecimiento. Buenos Aires: Amorrortu. 